\title{
“Triplet" polycistronic vectors encoding Gata4, Mef2c, and Tbx5 enhances postinfarct ventricular functional improvement compared with singlet vectors
}

\author{
Megumi Mathison, MD, PhD, ${ }^{a}$ Vivek P. Singh, $\mathrm{PhD},{ }^{a}$ Robert P. Gersch, PhD, ${ }^{b}$ Maricela O. Ramirez, BS, \\ Austin Cooney, PhD, ${ }^{a}$ Stephen M. Kaminsky, PhD, ${ }^{c}$ Maria J. Chiuchiolo, PhD, ${ }^{c}$ Ahmed Nasser, MD, \\ Jianchang Yang, MD, PhD, ${ }^{\mathrm{d}}$ Ronald G. Crystal, $\mathrm{MD},{ }^{\mathrm{c}}$ and Todd K. Rosengart, $\mathrm{MD}^{\mathrm{a}, \mathrm{e}}$
}

\begin{abstract}
Objective: The in situ reprogramming of cardiac fibroblasts into induced cardiomyocytes by the administration of gene transfer vectors encoding Gata4 (G), Mef2c (M), and Tbx5 (T) has been shown to improve ventricular function in myocardial infarction models. The efficacy of this strategy could, however, be limited by the need for fibroblast targets to be infected 3 times-once by each of the 3 transgene vectors. We hypothesized that a polycistronic "triplet" vector encoding all 3 transgenes would enhance postinfarct ventricular function compared with use of "singlet" vectors.
\end{abstract}

\begin{abstract}
Methods: After validation of the polycistronic vector expression in vitro, adult male Fischer 344 rats $(n=6)$ underwent coronary ligation with or without intramyocardial administration of an adenovirus encoding all 3 major vascular endothelial growth factor (VEGF) isoforms (AdVEGF-All6A positive), followed 3 weeks later by the administration to AdVEGF-All6A-positive treated rats of singlet lentivirus encoding $\mathrm{G}, \mathrm{M}$, or $\mathrm{T}\left(1 \times 10^{5}\right.$ transducing units each) or the same total dose of a GMT "triplet" lentivirus vector.
\end{abstract}

Results: Western blots demonstrated that triplet and singlet vectors yielded equivalent GMT transgene expression, and fluorescence activated cell sorting demonstrated that triplet vectors were nearly twice as potent as singlet vectors in generating induced cardiomyocytes from cardiac fibroblasts. Echocardiography demonstrated that GMT triplet vectors were more effective than the 3 combined singlet vectors in enhancing ventricular function from postinfarct baselines (triplet, $37 \% \pm 10 \%$; singlet, $13 \% \pm 7 \%$; negative control, $9 \% \pm 5 \% ; P<.05$ ).

Conclusions: These data have confirmed that the in situ administration of $\mathrm{G}, \mathrm{M}$, and $\mathrm{T}$ induces postinfarct ventricular functional improvement and that GMT polycistronic vectors enhance the efficacy of this strategy. (J Thorac Cardiovasc Surg 2014;148:1656-64)

Supplemental material is available online.

The studies by Takahashi and Yamanaka ${ }^{1}$ in 2006 demonstrating that genetic cellular reprogramming could

From the Division of Cardiothoracic Surgery, ${ }^{a}$ Michael E. DeBakey Department of Surgery, Baylor College of Medicine, Houston, Tex; Department of Surgery, ${ }^{b}$ Stony Brook University Medical Center, Stony Brook, NY; Department of Genetic Medicine, ${ }^{c}$ Weill Cornell Medical College, New York, NY; Department of Pathology, ${ }^{\mathrm{d}}$ Stony Brook University Medical Center, Stony Brook, NY; and the Department of Cardiovascular Surgery, ${ }^{\mathrm{e}}$ Texas Heart Institute, Houston, Tex.

This project was supported in part by a grant from James and Lisa Cohen and by the BCM Cytometry and Cell Sorting Core (the National Institutes of Health, P30AI036211, P30CA125123, S10RR024574), with funding from the National Institutes of Health (National Center for Research Resources grant S10RR024574, National Institute of Allergy and Infectious Diseases grant AI036211, and National Cancer Institute grant P30CA125123).

Disclosures: Authors have nothing to disclose with regard to commercial support.

Received for publication Jan 13, 2014; revisions received March 15, 2014; accepted

for publication March 21, 2014; available ahead of print April 19, 2014.

Address for reprints: Todd K. Rosengart, MD, Michael E. Division of Cardiothoracic

Surgery, Michael E. DeBakey Department of Surgery, Baylor College of Medicine,

1 Baylor Plaza, MS 390, Houston, TX 77030 (E-mail: todd.rosengart@bcm.edu).

0022-5223/\$36.00

Copyright (c) 2014 by The American Association for Thoracic Surgery

http://dx.doi.org/10.1016/j.jtcvs.2014.03.033 be used to generate induced pluripotent stem cells from adult somatic cells heralded a new era in tissue engineering. In 2010 , Ieda and colleagues ${ }^{2}$ advanced this work into the cardiac arena by demonstrating that a "cocktail" of transcription factors-Gata4 (G), Mef2c (M), and Tbx5 (T) - induced the conversion of cardiac fibroblasts directly into what they termed "induced cardiomyocyte" cells. This novel cell transdifferentiation strategy, subsequently used to genetically reprogram myocardial scar fibroblasts directly into cardiomyocyte-like cells in situ, offers the exciting new possibility of regenerating contractile tissue from otherwise unsalvageable infarcted myocardium. ${ }^{3-16}$ Such direct cardiac cellular reprogramming would obviate entirely the challenges of exogenous stem cell delivery or induced pluripotent stem staging strategies. ${ }^{17,18}$

Given that current cellular reprogramming strategies require the coincident infection of target cells by multiple gene transfer vectors, each encoding a single transgene, we hypothesized that use of a polycistronic "triplet" vector encoding all 3 of the requisite (GMT) transdifferentiating factors would be more efficient than the use of single transgene vectors. We further postulated that this enhanced 


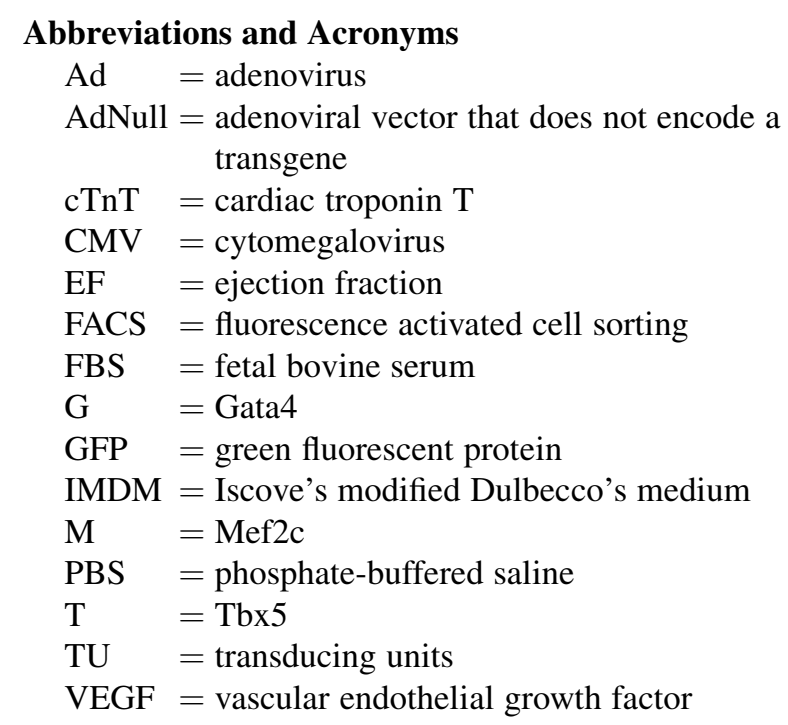

efficiency would result in improved postinfarct ventricular function compared with use of single transgene vectors. In the context of our previous demonstration that myocardial scar before vascularization enhances the efficacy of cardiac regeneration strategies, ${ }^{11}$ we have demonstrated in a rat coronary ligation infarct model after scar prevascularization with vascular endothelial growth factor (VEGF) that triplet GMT vectors induce greater improvements in postinfarct ventricular function than do equivalent total doses of singlet vectors.

\section{METHODS}

\section{Vectors and Cells}

An adenovirus vector (AdVEGF-All6A ${ }^{+}$) based on the human Ad5 serotype with E1a, partial E1b, E3 deletions that render the vector replication deficient was used to deliver all 3 major isoforms of VEGF $(121,165$, and 189$)$ to myocardial tissues. ${ }^{19}$ This AdVEGF-All6A ${ }^{+}$vector expression cassette, under the control of the cytomegalovirus (CMV) promoter or enhancer and the SV40 polyadenylation signal, incorporates a cDNA/genomic hybrid of human VEGF with intron modifications to allow expression of the 121, 165, and 189 alternative splicing VEGF isoforms. ${ }^{19}$ An adenoviral vector that does not encode a transgene (AdNull) was used as a control vector.

Lentivirus vectors were constructed to provide expression of GMT, with either "singlet" or "triplet" expression cassettes coupled to a green fluorescent protein (GFP) reporter transgene. Singlet GMT vectors were constructed, as previously described. ${ }^{11}$ In brief, the GMT transcription factor cDNAs (Table E1) were amplified from either commercially available sequences (Addgene) in the case of rat Gata4 (Genbank NM_144730) and rat Tbx5 (Genbank NM_001009964) or from rat heart tissue cDNA in the case of Mef2c (Genbank NM_001009964). Amplified sequences were cloned independently into the pENTR3C plasmid (Invitrogen, Life Technologies, Carlsbad, Calif). These plasmids were used to generate the singlet lentivirus vectors and as a template for assembling the triplet construct.

For the triplet construct, $\mathrm{G}, \mathrm{T}$, and $\mathrm{M}$ were amplified from the pENTR-Gata4, pENTR-Tbx5, and pENTR-Mef2c plasmids and assembled by overlapping polymerase chain reactions with $2 \mathrm{~A}$ self-processing viral peptide bridges downstream of a furin cleavage site (furin $2 \mathrm{~A}$ ) between each transcription factor gene. The Thosea asigna virus 2A sequence was used as a bridge between Gata4 and Tbx 5 proteins and the equine rhinitis A virus $2 \mathrm{~A}$ between the Tbx 5 and Mef2c sequences (Figure E1). The assembled GMT construct was cloned into the pENTR3C dual selection vector (Invitrogen) and incorporated into the FG12-CMV vector (Addgene), which includes the eGFP transgene under the control of the ubiquitin-C promoter for lineage efficiency analysis.

The F12-CMV-GMT plasmid containing the "triplet" expression cassette was co-transfected into the 293T human embryonic kidney cell line (ATCC, Manassas, Va), along with plasmids pMD2G and psPAX from the gateway system (Invitrogen) using lipofectamine 2000 reagent (Invitrogen). After 72 hours, supernatants containing virus were collected, clarified by centrifugation, and syringe filtered $(0.45-\mu \mathrm{m}$ pore size; Sarstedt, Nümbrecht, Germany). The virus was further pelleted from clarified supernatants by centrifugation for 2 hours at $10,000 \mathrm{~g}$ and resuspended in viral diluent $(3 \%$ sucrose, $10 \mathrm{mmol} / \mathrm{L}$ Tris- $\mathrm{HCl}, \mathrm{pH} 7.6$, $150 \mathrm{mmol} / \mathrm{L} \mathrm{NaCl}$ ).

To generate a control lentivirus vector, a homologous lentivirus vector with an expression cassette without a transgene but with an eGFP cassette under the control of the ubiquitin promoter was generated by co-transfecting the F12-CMV plasmid with the lentivirus packaging plasmids into $293 \mathrm{~T}$ cells, after which, the virus was purified as described in the preceding paragraphs.

Transduction units were established by quantitative polymerase chain reaction assay, as previously described. ${ }^{20}$ In brief, $1 \times 10^{5} 293 \mathrm{~T}$ cells were plated in a 24-well plate with $0.5 \mathrm{~mL}$ of Dulbecco's modified Eagle medium, $10 \%$ fetal bovine serum (FBS), polybrene $(8 \mu \mathrm{g} / \mathrm{mL})$, and $20 \mu \mathrm{L}$ of serially diluted lenti vector. After 48 hours in a carbon dioxide incubator, the cells were harvested, and the cellular DNA was purified using a DNA extraction kit (Qiagen, Venlo, Limburg, The Netherlands). Lenti RNA genome reverse transcribed into DNA was quantified using real time polymerase chain reaction and Stratagene Mx3005P (Life Technologies). SYBR Green SuperMix (Quanta Biosciences, Gaithersburg, Md) was used. The following primers located in the groupspecific antigen gene were used: $5^{\prime}$ - AGCGTCAGTATTAAGCGGGG- $3^{\prime}$ and $5^{\prime}$-AGGCCAGGATTAACTGCGA- $3^{\prime}$. The transducing unit was calculated as RNA genome/diploid cell $/ \mu \mathrm{L}$.

Adult cardiac fibroblasts were harvested from Fisher 344 adult male rats (weight 250-275 g; Harlan, Indianapolis, Ind) using protocols approved by the Baylor College of Medicine institutional animal care and use committee. These were prepared as described by Crabos and colleagues. ${ }^{20}$ In brief, the hearts excised from 2 to 3 rats were minced, washed in Hank's Balanced Salt Solution (Gibco, Life Technologies) supplemented with antibiotics (penicillin and streptomycin, $100 \mathrm{U} / \mathrm{mL}$ and $100 \mathrm{mg} / \mathrm{mL}$, respectively), and incubated at $37^{\circ} \mathrm{C}$ in the presence of $0.1 \%$ trypsin and $110 \mathrm{U} / \mathrm{mL}$ collagenase for 15 minutes. Disaggregated cells were serially pelleted, resuspended in Iscove's modified Dulbecco's medium (IMDM) with GLUT Max (Life Technologies) supplemented with $10 \%$ FBS and $1 \%$ penicillin and streptomycin antibiotics, and then seeded onto a $75-\mathrm{cm}^{2}$ flask and incubated in humidified $5 \%$ carbon dioxide $/ 95 \%$ air at $37^{\circ} \mathrm{C}$. After 120 minutes, the unattached cells were discarded, and the attached cells were cultured further in IMDM containing 10\% FBS for 4 days. The cells were then rinsed in phosphate-buffered saline (PBS) and harvested by enzymatic disaggregation $(0.05 \%$ trypsin), centrifuged, and resuspended in IMDM containing $10 \%$ FBS at a concentration of 100 cells $/ \mathrm{mL}$. The cells were then seeded onto 24 - or 6-well culture dishes and infected with the appropriate vectors after 48 hours.

\section{In Vitro Immunofluorescence Studies}

Triplet GMT lentivirus vectors $\left(9 \times 10^{5}\right.$ transducing units [TU]), singlet $\mathrm{G}, \mathrm{M}$, and $\mathrm{T}$ vectors $\left(9 \times 10^{5} \mathrm{TU}\right.$ each) or GFP control lentivirus vectors $\left(9 \times 10^{5} \mathrm{TU}\right)$ were added to rat cardiac fibroblast culture media (IMDM plus $10 \%$ FBS) for 48 hours in the presence of $1 \mu \mathrm{g} / \mathrm{mL}$ polybrene 

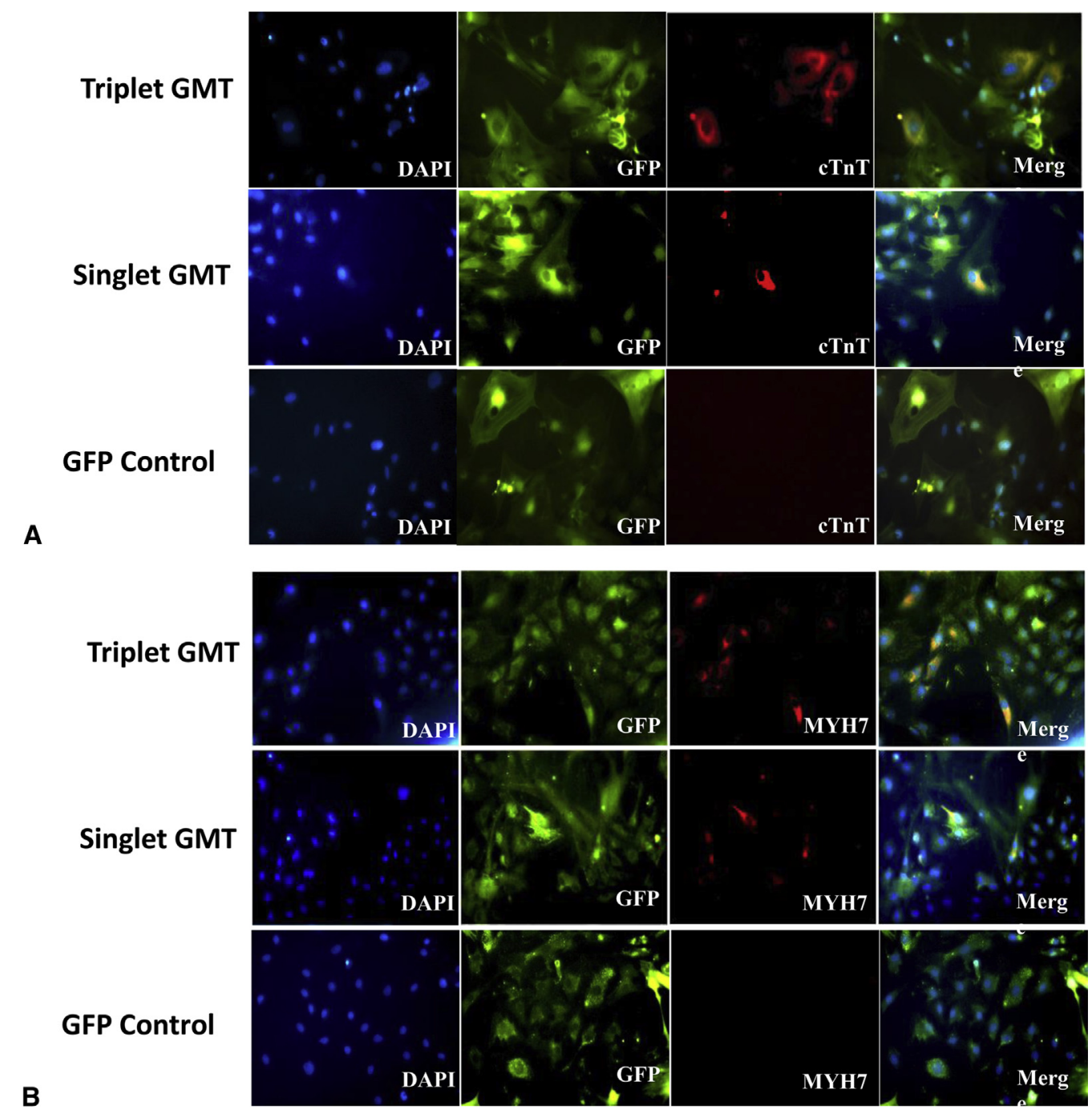

B

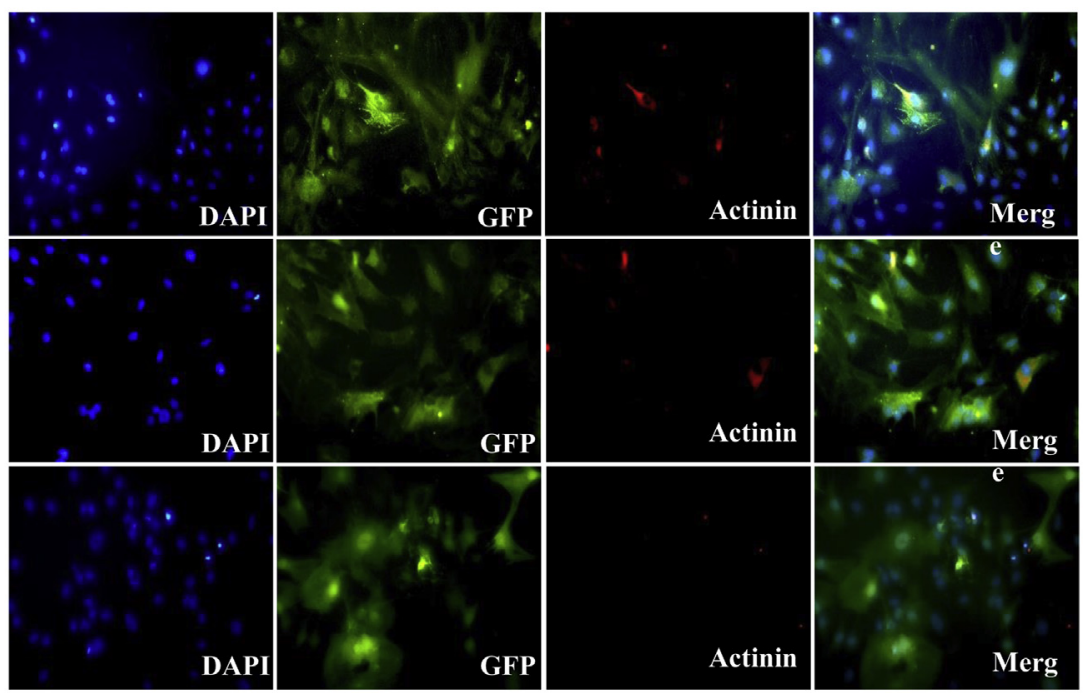

FIGURE 1. Immunohistochemistry. Fischer rat cardiac fibroblasts were infected with indicated viruses for 14 days, as described in the "Methods" section. The first column represents 4',6-diamidino-2-phenylindole (DAPI) staining to identify cell nuclei. The second column represents green fluorescent protein 
(EMD Millipore, Billerica, Mass). This medium was then removed, and the cells were allowed to transdifferentiate under normal culture conditions for 14 days. The cells were then washed twice with PBS and fixed with $4 \%$ paraformaldehyde (Affymetrix, Santa Clara, Calif) for 10 minutes. The cells were then permeabilized with $0.5 \%$ Saponin (Sigma-Aldrich, St Louis, Mo) at room temperature for 10 minutes. The slides were blocked with $10 \%$ goat serum (Santa Cruz Biotechnology, Santa Cruz, Calif) before incubation in $5 \%$ goat serum with primary antibodies directed toward cardiac troponin $\mathrm{T}$ (Abcam, Cambridge, UK), $\beta$-myosin heavy chain 7 (Abcam), or $\alpha$-sarcomeric actinin (Abcam). Primary antibodies were labeled with fluorescent secondary $(647 \mathrm{~nm}$; Alexa Fluor, Invitrogen), and fluorescence was visualized using a Ti-S inverted phase/fluorescent microscope with an SPOT cooled 2.0-megapixel digital microscope camera system (Nikon, Tokyo, Japan).

\section{Fluorescence Activated Cell Sorting}

Cells treated as described above were washed with PBS and treated with $0.05 \%$ trypsin (Gibco). The cells were then pelleted and permeabilized with $0.1 \%$ saponin for $10 \mathrm{~min}$ at room temperature, re-pelleted and suspended in 5\% goat serum, then incubated with anti-cTnT primary antibody (Abcam) followed by incubation with a fluorescent secondary antibody (647 nm; Alexa Fluor). The cells were then fixed with $1 \%$ paraformaldehyde and analyzed for fluorescence using fluorescence activated cell sorting (FACS) Diva software, version 6.0, on a FACSCalibur flow cytometer (Becton Dickinson, Franklin Lakes, NJ).

\section{Rat Coronary Artery Ligation Myocardial Infarction Model}

Myocardial infarction was created in adult male Fisher 344 rats (weight, 275-300 g; Harlan), using techniques standard to our laboratory. ${ }^{11,21,22}$ The Stony Brook University institutional animal care and use committee approved this protocol. The rats were housed, operated on, and cared for in facilities run by the Division of Laboratory Animal Resources at Stony Brook University, which is fully accredited by the Association for the Assessment and Accreditation of Laboratory Animal Care International.

Three weeks after initial coronary ligation and direct myocardial administration of AdVEGF-All6A ${ }^{+}\left(1 \times 10^{9}\right)$ or an equivalent dose of AdNull, the rats underwent a second thoracotomy and injection (5 uniformly distributed $20-\mu \mathrm{L}$ aliquots; Figure E2) into the infarct zone of a cocktail of singlet lentiviruses each encoding G, M, or T $\left(1 \times 10^{5}\right.$ TU of each vector) or the same total dose $\left(3 \times 10^{5} \mathrm{TU}\right)$ of a GMT "triplet" lentivirus vector ( $n=6$ /group). The rats were allowed to recover and then euthanized 4 weeks later by deep $(4 \%)$ isoflurane anesthesia followed by exsanguination, consistent with the American Veterinary Medical Association guidelines.

\section{Echocardiography}

Echocardiography was performed with the rats under light anesthesia with 3\% isoflurane using a Veno 770 Imaging System (VisualSonics, Inc, Toronto, Ontario, Canada), as previously described. ${ }^{11,21,22}$ Echocardiographic images were obtained in the parasternal long-axis and short-axis views at specified points by investigators who were unaware of the treatment groups. The left ventricular end-systolic and end-diastolic diameters were measured from the M-mode tracings. The change in the ejection fraction (EF) from baseline was calculated as [(EF at day 35 [2 weeks after GMT administration]) - (EF at day 21 [point of GMT administration])]/(EF at day 21) and [(EF at day 49 [4 weeks after GMT administration] $)-($ EF at day 21$)] /($ EF at day 21$)$

\section{Histologic Examination}

To obtain the cardiac tissue specimens, the rats were exsanguinated under deep anesthesia by an incision made in the right atrium. With the heart still beating, it was perfused with normal saline and fixed with PBS (pH 7.2) containing 4\% (wt/vol) paraformaldehyde by way of a 25 -gauge needle inserted into the left ventricular apex. The heart was then harvested and rinsed with saline to clear the blood. The excised hearts were fixed with $4 \%$ paraformaldehyde for 24 hours and then with $2 \%$ paraformaldehyde for 48 hours at $4^{\circ} \mathrm{C}$. The heart was then cut transversally and sectioned, with 2 slices $(2-3 \mathrm{~mm})$ obtained, 1 immediately cephalad and 1 immediately caudal to the transverse centerline of the infarct region, which was readily identifiable by gross inspection. After paraffin embedding of these slices, four 5 - $\mu \mathrm{m}$-thick sections were obtained at $100-\mu \mathrm{m}$ intervals.

To assess the extent of fibrosis, 8 sections per rat (at $100-\mu \mathrm{m}$ intervals between each section) obtained as described were stained with Masson's trichrome. The fibrotic area (blue) and the nonfibrotic region (red) were outlined using Adobe Photoshop CS5 software (Adobe, San Jose, Calif) and then quantified using MATLAB and Simulink software (MathWorks, Inc, Natick, Mass). The percentage of fibrosis was calculated as follows: ([blue pixels/blue + red pixels $] \times 100$ ). In each rat, the greatest value for the percentage of fibrosis in the 8 slides was designated as the percentage of fibrosis for the rat.

For myofibroblast identification, the histologic section for each rat demonstrating the greatest cross-sectional area of fibrosis, identified as described, was stained for $\alpha$-smooth muscle actin (anti-actin smooth muscle, Spring Bioscience). $\alpha$-Smooth muscle actin-positive cells exclusive of those found in vascular structures or endocardium were counted at $200 \times$ magnification.

\section{Statistical Analysis}

Statistical analysis was performed using STATA, version 13 (StataCorp, College Station, Tex). The data are presented as the mean \pm standard error of the mean. For analyses of $\alpha$-smooth muscle actin/histologic section, after obtaining statistical significance for the Shapiro-Wilk $W$ test $(P=.003)$ and the Bartlett's test $(P<.01)$, no additional analysis was performed after obtaining a nonsignificant Kruskal-Wallis test $(P=.08)$. For an assessment of the extent of fibrosis, although the results of the Shapiro-Wilk W test was not significant $(P=.19)$, the results from the Bartlett test was significant $(P<.01)$; however, no additional analysis was performed after a nonsignificant Kruskal-Wallis test. For analyses of echocardiographic assessment of the mean of the change in EF for each rat from the interval from lentivirus administration to assessment 4 weeks later, the $P$ values were as follows: Shapiro-Wilk W, $P=.14$; Bartlett test, $P=.38$; and Levene's test, $P=.11$. Therefore, a 1-way analysis of variance test was applied to the data. Because the analysis of variance test was significant $(P<.0001)$, additional analysis of variance tests with Bonferroni's correction were performed for pair comparisons, as reported in the "Results" section.

$(G F P)$ fluorescence to identify cells infected by lentivirus vectors. The third column represents red staining of relevant cardiomyocyte markers (Top, cardiac troponin T [cTnT]; Middle, myosin heavy chain 7; Bottom, $\alpha$-sarcomeric actinin). The fourth column depicts a merge of the previous 3 images. Note coincidence of these respective markers and binucleated cells typical of cardiomyocytes and that GFP-negative cells failed to express these markers. Uninfected rat cardiac fibroblasts did not express either marker (data not shown). All photomicrographs were taken at $\times 400$ magnification. GMT, Gata4, Mef2c, and Tbx5; MYH7, myosin heavy chain 7. 


\section{RESULTS \\ In Vitro Studies}

The competency of the triplet GMT expression vector was confirmed by in vitro cell infection followed 3 days later with Western analysis, which demonstrated expression of all 3 of the reprogramming transcription factors at expression levels equivalent to those of the singlet vectors (Figure E3). Confirmation of the ability of these transcription factors to induce cellular transdifferentiation, distinguished by expression of selected markers of a cardiomyocyte-like phenotype, was obtained by immunofluorescence staining of rat cardiac fibroblasts 14 days after triplet or singlet GMT lentiviral transduction. In these studies, cells exposed to triplet or singlet GMT vectors expressed the cardiomyocyte-specific markers cardiac troponin T (cTnT), $\beta$-myosin heavy chain 7 , and $\alpha$-sarcomeric actinin. However, fibroblasts infected with the control vectors or assayed without the primary cTnT antibodies failed to demonstrate expression of these markers (Figure 1, $A-C$ ). Consistent with the increased density of cardiomyocyte marker staining noted after triplet versus singlet GMT infections in these immunofluorescence studies, FACS quantification demonstrated that cTnT expression by cardiac fibroblasts 14 days after exposure to triplet GMT vectors was nearly double that of the fibroblasts exposed to ( 3 times the total viral dose of) singlet vectors (Figure 2).

\section{Histologic Assessment of GMT-Treated, Postinfarct Ventricles}

Although they did not reach statistical significance, decreases in the total area of fibrosis and the number of scar-producing myofibroblasts were observed 4 weeks after GMT triplet versus singlet vector administration in a rat myocardial infarction model compared with negative controls (Figure 3,A). The maximum cross-sectional area of fibrosis detected in the sections of infarct zones was $21 \% \pm 1 \%, 26 \% \pm 3 \%$, and $31 \% \pm 6 \%$ for triplet, singlet, and GFP control lentivirus-treated rats, respectively (Figure 3, B). Consistent with these findings, the mean number of myofibroblasts detected in triplet, singlet, and GFP control lentivirus-treated rats was $39 \pm 12,63 \pm$ 8 , and $108 \pm 31$ cells/section, respectively (Figure 3, C). The mean number of myofibroblasts/section detected in the GFP control lentivirus group was significantly greater $(P<.05)$ than the average of $52 \pm 8$ myofibroblasts/section detected for the 2 GMT-treated groups analyzed together.

\section{Improvement in Ventricular Function After GMT Triplet Versus Singlet Administration}

Three-dimensional echocardiography demonstrated that the ventricular ejection fraction (EF) after coronary ligation was reduced by $\approx 35 \%$ from the baseline values (Table 1). This decrease in cardiac function had persisted 3 weeks later, at GMT or GFP control lentivirus administration. As depicted in Figure 4, the mean change in the EF for each rat from the lentivirus administration to the follow-up echocardiogram 4 weeks later was significantly greater $(P<.05)$ in the triplet GMT group $(37 \% \pm 10 \%)$ compared with the singlet GMT group $(13 \% \pm 7 \%)$ or AdNull/GFP controls $(-9 \% \pm$ $5 \%)$.

\section{DISCUSSION}

The present study has demonstrated that polycistronic "triplet" vectors encoding a complete set of cardiac cellular reprogramming transcription factors (Gata4, Mef2c, and Tbx5), first identified by Ieda and colleagues, ${ }^{2}$ are more efficient than a cocktail of "singlet" vectors, each expressing only 1 of these transgenes, in transdifferentiating cardiac fibroblasts into a cardiomyocyte-like cell in vitro expressing a number (although not necessarily all) of the features typical of a cardiomyocyte. Our study also demonstrated that use of polycistronic cellular reprogramming vectors enhances postinfarct ventricular function to an extent greater than that obtained with equivalent total viral dosages of singlet vectors.

The mechanism limiting the efficiency of transdifferentiation is unknown but might be linked to the number and/or choice of reprogramming factors and the responsiveness of the target cells to transdifferentiation. Although a few studies have reported transdifferentiating efficiencies $>20 \%$ achieved with the addition of an increased number of, or different, reprogramming transgenes than the "standard" GMT cocktail also used in the present study, most reported transdifferentiation efficiency rates have been in the $5 \%$ to $10 \%$ range..$^{2-16,23}$ Our demonstration of nearly double the rate of cardiomyocyte marker expression in vitro with use of a triplet GMT vector compared with the use of 3 times the total dose of singlet vectors supports the use of polycistronic vectors as 1 component of a strategy to enhance the efficiency of cellular transdifferentiation.

One additional scientific advantage to the use of polycistronic vectors is their potential value in deciphering the genetic mechanisms leading to (or blocking) cellular reprogramming. In contrast to previous singlet vector studies that were difficult to interpret regarding whether the cells had been effectively infected with 1 , 2 , or all $3 \mathrm{G}, \mathrm{M}$, and $\mathrm{T}$ vectors, the use of a polycistronic vector permits the identification of GFP-expressing cells infected with the GMT polycistronic vector and expressing all 3 reprogramming transgenes. In our analysis, we observed that almost 3 of 4 cells expressing GFP after triplet vector exposure did not express cTnT. Future studies can help to elucidate the genetic basis of 

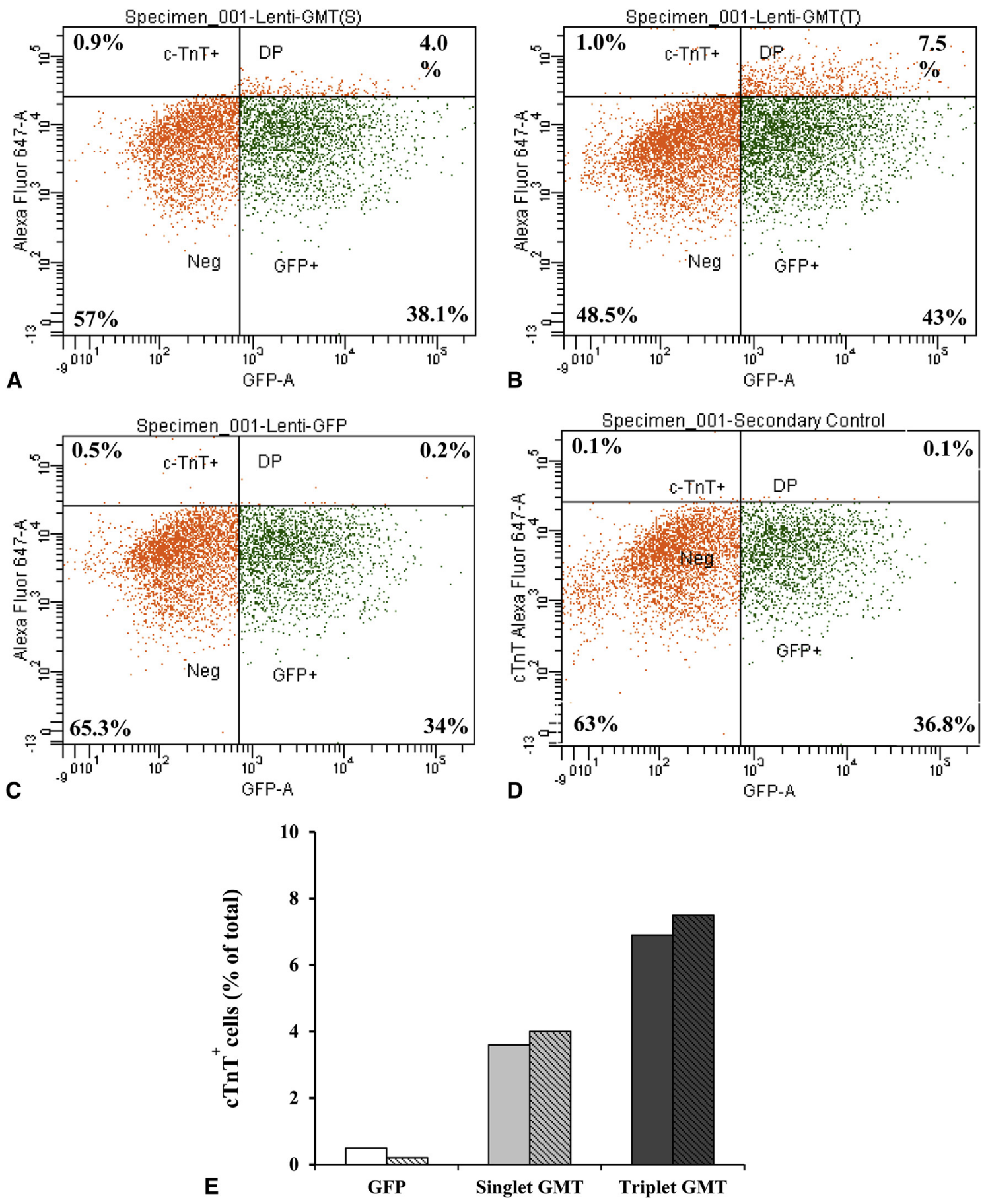

FIGURE 2. Fluorescence activated cell sorting (FACS) analysis. A-D, Representative flow cytometry plots for analysis of cardiac troponin T-positive $(c T n T+)$ cells 14 days after infection of rat cardiac fibroblasts (RCFs) with singlet, triplet, or green fluorescent protein $(G F P)$ control lentivirus. The numbers of the upper right panel (double positive $[D P]$ ) indicate the percentage of GFP $+/ \mathrm{cTnT}+$ cells. A, RCFs infected with singlet Gata4, Mef2c, and Tbx5 $(G M T)$, demonstrating $4 \%$ expression of cTnT in GFP + cells. B, RCFs infected with triplet GMT, demonstrating $7.5 \%$ expression of cTnT in GFP + cells. C, RCFs infected with GFP control lentivirus. D, RCFs infected with GMT, with use of secondary antibody only. Plots show a minimum of 10,000 events. E, A summary of the flow cytometry analysis of 2 separate experiments. The percentage of cTnT + cells after infection of RCFs with singlet GMT, triplet GMT, or GFP control lentivirus.

why such GMT-expressing "meso" cells were not more completely converted to a cardiomyocyte-like phenotype and better understand the features that are and are not present in these cells and how potential observed roadblocks can be addressed to enhance transdifferentiation. 


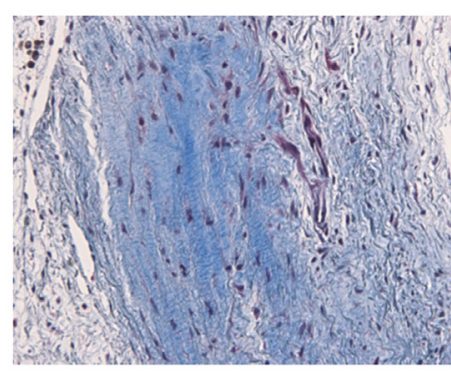

AdNull/GFP

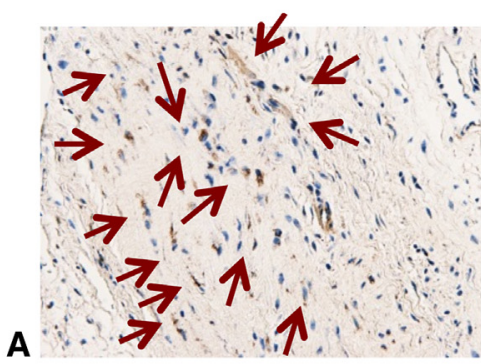

AdNull/GFP

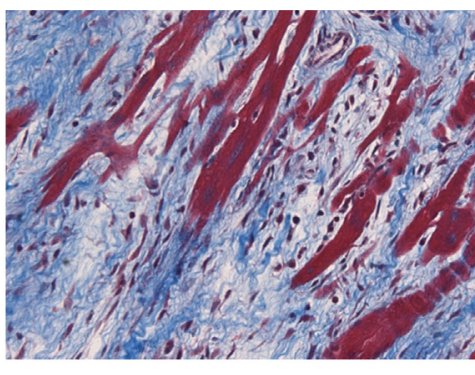

VEGF/Singlet GMT

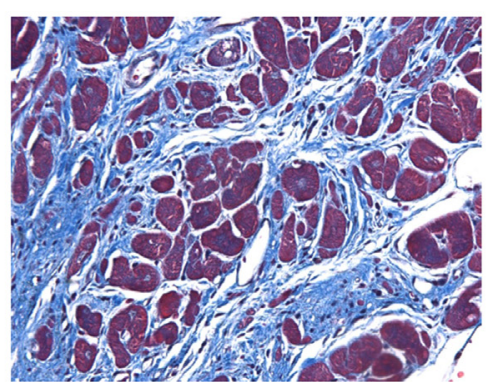

VEGF/Triplet GMT

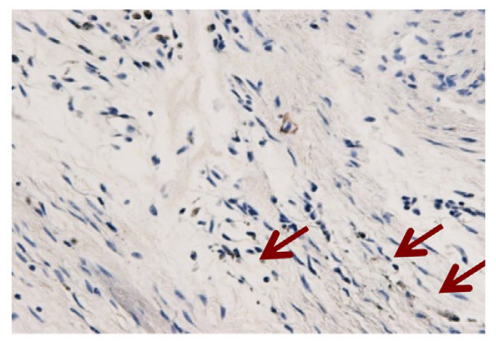

VEGF/Singlet

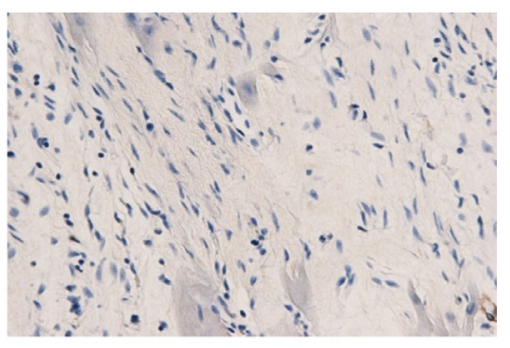

VEGF/Triplet GMT
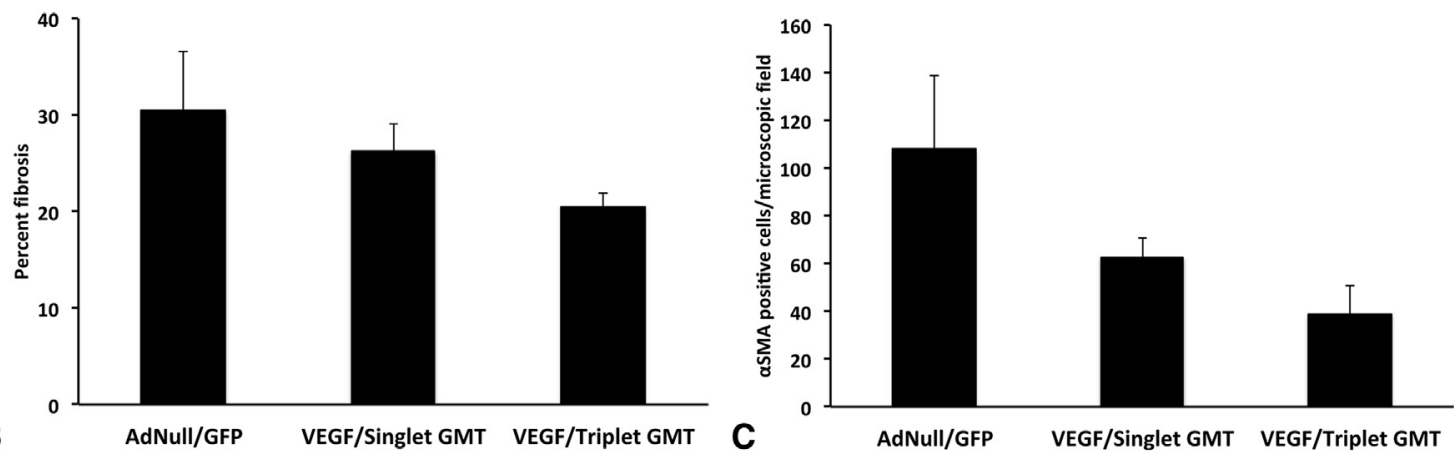

FIGURE 3. Extent of fibrosis. Fibrosis and myofibroblast density determined from myocardial tissue harvested 7 weeks after coronary ligation and administration of AdVEGF-All6A+ or the control vector AdNull and 4 weeks after administration of lentivirus encoding singlet or triplet Gata4, Mef2c, and Tbx5 (GMT) or a green fluorescent protein (GFP) control lentivirus, as determined in the "Methods" section. A, Photomicrographs of representative sections (original magnification $\times 200$ ). The top images depict the extent of fibrosis as stained by Masson-Trichrome. The bottom row depicts myofibroblasts (arrows) stained by $\alpha$-smooth muscle actin. Left, AdNull/GFP; Center, AdVEGF-All6A+/singlet GMT; Right, AdVEGF-All6A+/triplet GMT. B, Extent of fibrosis for the 3 treatment groups identified by Masson-Trichrome staining. C, Myofibroblasts identified per microscopic field by nonvascular $\alpha$-smooth muscle actin $(\alpha S M A)$ staining of the infarct and border zones of the sections. AdNull, Adenoviral vector that does not encode a transgene; $V E G F$, vascular endothelial growth factor.

In contrast, given the in vitro delivery of a theoretically equivalent "dose" of G, M, and T transgenes in the present study, either by singlet or triplet vectors (ie, $9 \times 10^{5} \mathrm{TU}$ of a triplet vector encoding GMT or $9 \times 10^{5} \mathrm{TU}$ of each of the G, $\mathrm{M}$, and $\mathrm{T}$ singlet vectors), it is interesting to speculate why the polycistronic vector is more effective than the singlet vector cocktails in inducing transdifferentiation in vitro. One possibility is simply that integration or expression of a singlet vector inhibits the expression of other vectors-a handicap theoretically overcome with use of a triplet expression vector. However, the similar levels of GFP expression noted in the singlet versus triplet-infected cells despite the use of triple the total vector dose in the former experimental group suggested that integration of 1 singlet vector might represent a barrier to additional viral integration. Additional studies of this phenomenon would require use of markers specific to each of the singlet vectors.

$\mathrm{We}$, and others, have previously provided evidence that the improvements in ventricular function observed in animals receiving reprogramming agents could have resulted from the observed increase in the generation of cardiomyocyte-like cells in the infarct territories. ${ }^{6,7,10,11}$ We did not repeat the in vivo cardiomyocyte marker analyses, including those of contractile function, or 
TABLE 1. Cardiac functional index at various measurement points

\begin{tabular}{lccccc}
\hline \multicolumn{1}{c}{ Parameter } & \multicolumn{2}{c}{ Interval from coronary ligation (d) } & F statistic; associated $\boldsymbol{P}$ value* \\
\hline Ejection fraction & 0 & 3 & $21 \dagger$ & 35 & 49 \\
AdVEGF/GMT triplet & $76 \pm 2$ & $37 \pm 2$ & $36 \pm 3$ & $45 \pm 3$ & $48 \pm 3$ \\
AdVEGF/GMT singlet & $75 \pm 1$ & $34 \pm 1$ & $38 \pm 1$ & $34 \pm 1$ & $43 \pm 2$ \\
AdNull/GFP & $76 \pm 1$ & $41 \pm 2$ & $43 \pm 2$ & $40 \pm 3$ & $39 \pm 3$ \\
End-diastolic volume & & & & \\
AdVEGF/GMT triplet & $285 \pm 11$ & $313 \pm 10$ & $427 \pm 59$ & $392 \pm 79$ & $290 \pm 33$ \\
AdVEGF/GMT singlet & $294 \pm 10$ & $429 \pm 35$ & $482 \pm 58$ & $382 \pm 20$ & $434 \pm 46$ \\
AdNull/GFP & $250 \pm 10$ & $323 \pm 8$ & $314 \pm 19$ & $337 \pm 20$ & $360 \pm 39$ \\
End-systolic volume & & & & \\
AdVEGF/GMT triplet & $68 \pm 7$ & $195 \pm 3$ & $279 \pm 45$ & $222 \pm 58$ & $153 \pm 26$ \\
AdVEGF/GMT singlet & $75 \pm 3$ & $286 \pm 23$ & $304 \pm 43$ & $253 \pm 15$ & $251 \pm 33$ \\
AdNull/GFP & $59 \pm 3$ & $190 \pm 8$ & $179 \pm 17$ & $203 \pm 19$ & $218 \pm 30$ \\
Fractional shortening & & & & \\
AdVEGF/GMT triplet & $46 \pm 2$ & $19 \pm 1$ & $18 \pm 2$ & $24 \pm 2$ & $25 \pm 2$ \\
AdVEGF/GMT singlet & $45 \pm 1$ & $17 \pm 1$ & $19 \pm 1$ & $17 \pm 1$ & $22 \pm 2$ \\
AdNull/GFP & $46 \pm 1$ & $21 \pm 1$ & $22 \pm 1$ & $20 \pm 2$ & $20 \pm 2$ \\
\hline AdVEGF, Adenovirus vascular endothelial growth factor; GMT, Gata4, Mef2c, and Tbx5; AdNull, adenoviral vector that does not encode a transgene; GFP, green fluorescent \\
protein. *For repeated measure analysis of variance; results shown indicate treatment $\times$ time interaction effect. $\dagger$ Point of GMT or GFP control lentivirus vector delivery.
\end{tabular}

perform cell lineage analyses in the present report. Thus, it is conceivable that the observed outcomes resulted from paracrine or other effects distinct from the cell transdifferentiation properties of GMT. Therefore, although the decreases in fibrosis in each of the 2 GMT-treated groups in the present study did not reach statistical significance, the trend toward a decrease in fibrosis observed in the present study in the GMT-treated versus control rats is consistent with our previous observations. ${ }^{11}$

We also did not in the present study repeat our previous experiments demonstrating the importance of prevascularization scarring in enhancing the effects of GMT that could exceed the effects of VEGF alone. ${ }^{11}$

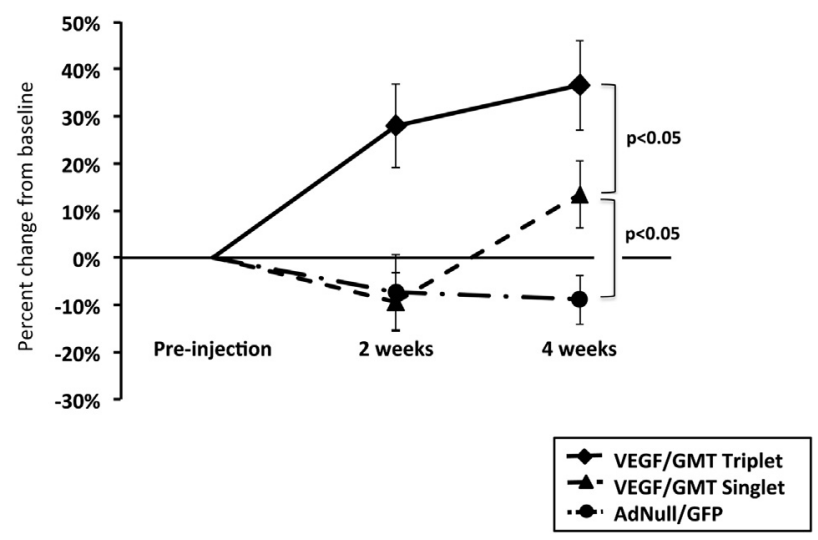

FIGURE 4. Echocardiography analysis showing the mean change in ejection fraction for each rat from lentivirus administration (day 21) at baseline to the follow-up echocardiogram 2 (day 35) and 4 (day 49) weeks later. VEGF, Vascular endothelial growth factor; $G M T$, Gata4, Mef2c, and Tbx5; AdNull, adenoviral vector that does not encode a transgene; $G F P$, green fluorescent protein.
Therefore, just as in our previous studies, we could not rule out an effect of VEGF or GMT in these studies, independent of the presumed transdifferentiation effects. However, our previous exogenous stem cell delivery studies suggested that such prevascularization is important to supporting the survival of high-metabolic (endogenous or exogenous) cells. ${ }^{21,22}$ Likewise, because we did not repeat previous in vivo cell lineage studies to confirm the generation of cardiomyocyte-like cells from fibroblasts in vivo, ${ }^{2,4,7,10,12}$ these previous studies and our own in vitro results suggest this as only 1 possible mechanism underlying the improvements in ventricular function observed in vivo. Our present findings have confirmed previous observations of a potentially important role for GMT in decreasing fibrosis-generating myofibroblast density and fibrosis in a mechanism underlying the observed improvements in ventricular function. ${ }^{6,7,11}$ The ability of lentivirus to infect dividing and nondividing cells, in contrast to a previous polycistronic vector study using a retrovirus that induces transgene expression only in dividing cells, ${ }^{10}$ also leaves open the as yet unexplored possibility of a locus of action for GMT involving native cardiomyocytes and fibroblasts. However, these target cells were not specifically identified in the present study. Each of these theoretical mechanisms, potentially involving paracrine mechanisms of action, requires additional investigation.

\section{CONCLUSIONS}

The results from the present study have advanced previous cellular reprogramming investigations by demonstrating that polycistronic vectors can be used to enhance the efficiency of cell transdifferentiation in vitro. This strategy, resulting in improvements in postinfarct 
ventricular function in vivo, could represent an important new method of regenerating functional myocardium from postinfarct scar tissue as a potentially clinically relevant method of treating patients with end-stage heart disease. Although we elected to use relatively low viral titers in our studies to be consistent with other published reports, our in vitro studies using even greater vector dosages without resultant cytotoxicity suggest that the use of greater vector dosages than those used in the present study might yield even greater improvements in vivo.

The improvements in cellular transdifferentiation efficiency demonstrated with the use of polycistronic vectors in vitro could prove to be highly consequential given the likely regulatory hurdle for evaluating each of the 3 vectors separately should multiple vectors be required for clinical trials and the relative resistance of human cells to transdifferentiation that can be anticipated in the clinical setting. It has already recently been demonstrated that cocktails of $\leq 4$ or 5 transgenes are needed to generate induced cardiomyocytes from human cells, which appear to be more refractory to transdifferentiation than rodent cells. ${ }^{12,15,16}$

We thank Courtney Nalty, MSPH, for her help with the statistical analysis.

\section{References}

1. Takahashi K, Yamanaka S. Induction of pluripotent stem cells from mouse embryonic and adult fibroblast cultures by defined factors. Cell. 2006;126: 663-76.

2. Ieda M, Fu JD, Delgado-Olguin P, Vedantham V, Hayashi Y, Bruneau BG, et al. Direct reprogramming of fibroblasts into functional cardiomyocytes by defined factors. Cell. 2010;142:375-86.

3. Passier R, Mummery C. Getting to the heart of the matter: direct reprogramming to cardiomyocytes. Cell Stem Cell. 2010;7:139-41.

4. Efe JA, Hilcove S, Kim J, Zhou H, Ouyang K, Wang G, et al. Conversion of mouse fibroblasts into cardiomyocytes using a direct reprogramming strategy. Nat Cell Biol. 2011;13:215-22.

5. Jayawardena TM, Egemnazarov B, Finch EA, Zhang L, Payne JA, Pandya K, et al. MicroRNA-mediated in vitro and in vivo direct reprogramming of cardiac fibroblasts to cardiomyocytes. Circ Res. 2012;110:1465-73.

6. Qian L, Huang Y, Spencer CI, Foley A, Vedantham V, Liu L, et al. In vivo reprogramming of murine cardiac fibroblasts into induced cardiomyocytes. Nature. 2012;485:593-8.
7. Song K, Nam Y, Luo X, Qi X, Tan W, Huang GN, et al. Heart repair by reprogramming non-myocytes with cardiac transcription factors. Nature. 2012; 485:599-604.

8. Srivastava D, Ieda M. Critical factors for cardiac reprogramming. Circ Res. 2012; 111:5-8.

9. Protze S, Khattak S, Poulet C, Lindemann D, Tanaka EM, Ravens U. A new approach to transcription factor screening for reprogramming of fibroblasts to cardiomyocyte-like cells. J Mol Cell Cardiol. 2012;53:323-32.

10. Inagawa K, Miyamoto K, Yamakawa H, Muraoka N, Sadahiro T, Umei T, et al. Induction of cardiomyocyte-like cells in infarct hearts by gene transfer of Gata4, Mef2c, and Tbx5. Circ Res. 2012;111:1147-56.

11. Mathison M, Gersch RP, Nasser A, Lilo S, Korman M, Fourman M, et al. In vivo cardiac cellular reprogramming efficacy is enhanced by angiogenic preconditioning of the infarcted myocardium with vascular endothelial growth factor. J Am Heart Assoc. 2012;1:e005652.

12. Nam Y, Song K, Luo X, Daniel E, Lambeth K, West K, et al. Reprogramming of human fibroblasts toward a cardiac fate. Proc Natl Acad Sci U S A. 2013;110: 5588-93.

13. Christoforou N, Chellappan M, Adler AF, Kirkton RD, Wu T, Addis RC, et al. Transcription factors MYOCD, SRF, Mesp1 and SMARCD3 enhance the cardio-inducing effect of Gata4, Tbx5, and Mef2c during direct cellular reprogramming. PLoS One. 2013;8:e63577.

14. Addis RC, Ifkovits JL, Pinto F, Kellam LD, Esteso P, Rentschler S, et al. Optimization of direct fibroblast reprogramming to cardiomyocytes using calcium activity as a functional measure of success. J Mol Cell Cardiol. 2013; 60:97-106

15. Wada R, Muraoka N, Inagawa K, Yamakawa H, Miyamoto K, Sadahiro T, et al. Induction of human cardiomyocyte-like cells from fibroblasts by defined factors. Proc Natl Acad Sci U S A. 2013;110:12667-72.

16. Fu J, Stone NR, Liu L, Spencer I, Qian L, Hayashi Y, et al. Direct reprogramming of human fibroblasts toward a cardiomyocyte-like state. Stem Cell Reports. 2013; $1: 235-47$

17. Mummery C. Induced pluripotent stem cells-a cautionary note. $N$ Engl J Med. 2011;364:2160-2.

18. Rosenzweig A. Cardiac cell therapy-mixed results from mixed cells. $N$ Engl J Med. 2006;355:1274-7.

19. Amano H, Hackett NR, Kaner RJ, Whitlock P, Rosengart TK, Crystal RG Alteration of splicing signals in a genomic/cDNA hybrid VEGF gene to modify the ratio of expressed VEGF isoforms enhances safety of angiogenic gene therapy. Mol Ther. 2005;12:716-24.

20. Crabos M, Roth M, Hahn AW, Erne P. Characterization of angiotensin II receptors in cultured adult rat cardiac fibroblasts: coupling to signaling systems and gene expression. J Clin Invest. 1994;93:2372-8.

21. Retuerto MA, Schalch P, Patejunas G, Carbray J, Liu N, Esser K, et al. Angiogenic pretreatment improves the efficacy of cellular cardiomyoplasty performed with fetal cardiomyocyte implantation. J Thorac Cardiovasc Surg 2004;127:1041-9.

22. Retuerto MA, Beckmann JT, Carbray J, Patejunas G, Sarateanu S, Kane B, et al. Angiogenic pretreatment enhances myocardial function following cellular cardiomyoplasty with skeletal myoblasts. J Thorac Cardiovasc Surg. 2007;133:478-84.

23. Hirai H, Katoku-Kikyo N, Keirstead SA, Kikyo N. Accelerated direct reprogramming of fibroblasts into cardiomyocyte-like cells with the MyoD transactivation domain. Cardiovasc Res. 2013;100:105-13. 


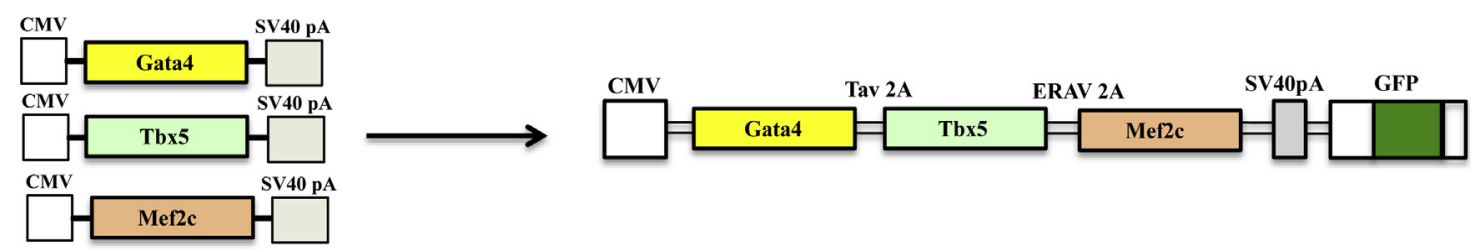

FIGURE E1. Schematic representation of the triplet Gata4, Mef2c, and Tbx5 (GMT) expression cassette. The 3 transcription factor transgenes were incorporated into a single open reading frame under the control of the human cytomegalovirus $(C M V)$ immediate or early promoter and a Simian virus 40 (SV40) polyadenylation signal and expressed as a self-cleavable construct that included the Thosea asigna virus (Tav) 2A self-cleavable sequence and the Equine rhinitis A virus (ERAV) 2A sequence. GFP, Green fluorescent protein.

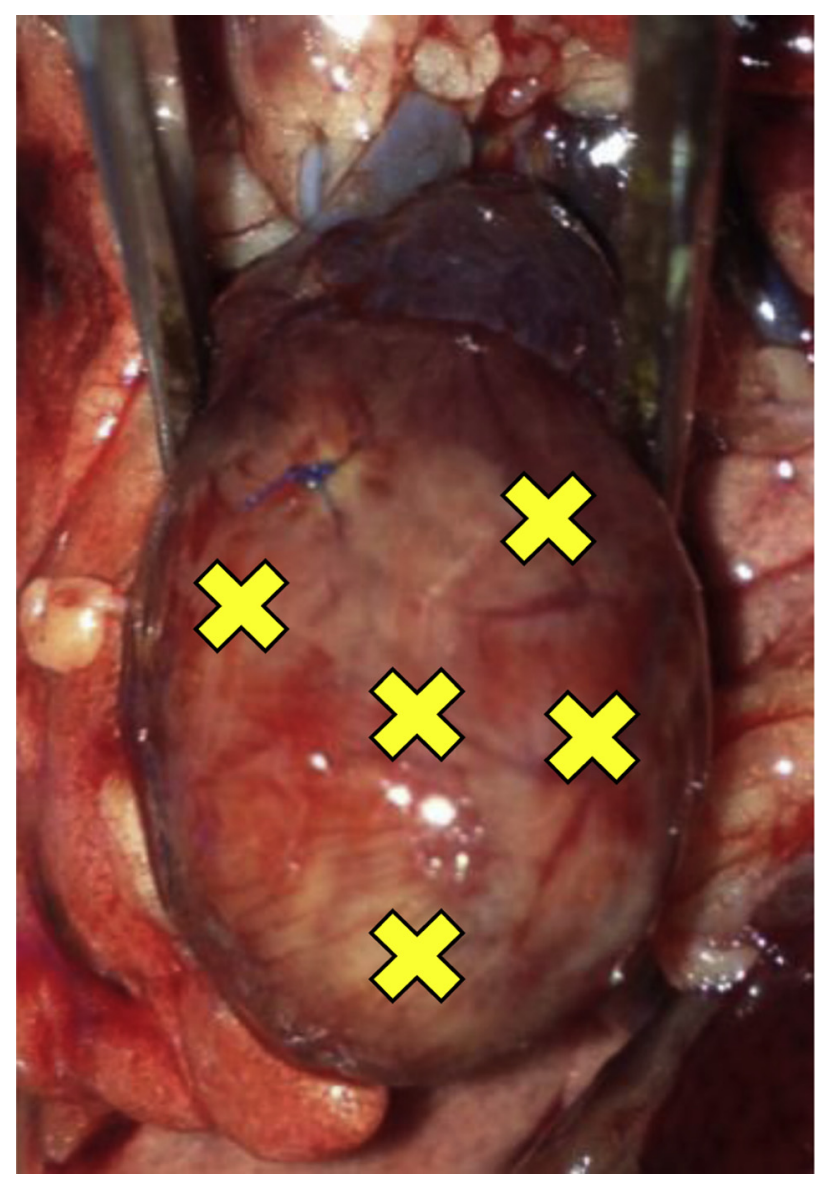

FIGURE E2. Depiction of vector administration. At proximal left anterior descending coronary artery ligation (site identified by blue polypropylene suture near the base of the heart) and 3 weeks later, the vector was administered by direct myocardial injection (5 uniformly distributed $20-\mu \mathrm{L}$ aliquots [yellow crosses]) around the periphery and at the center of the infarct zone.

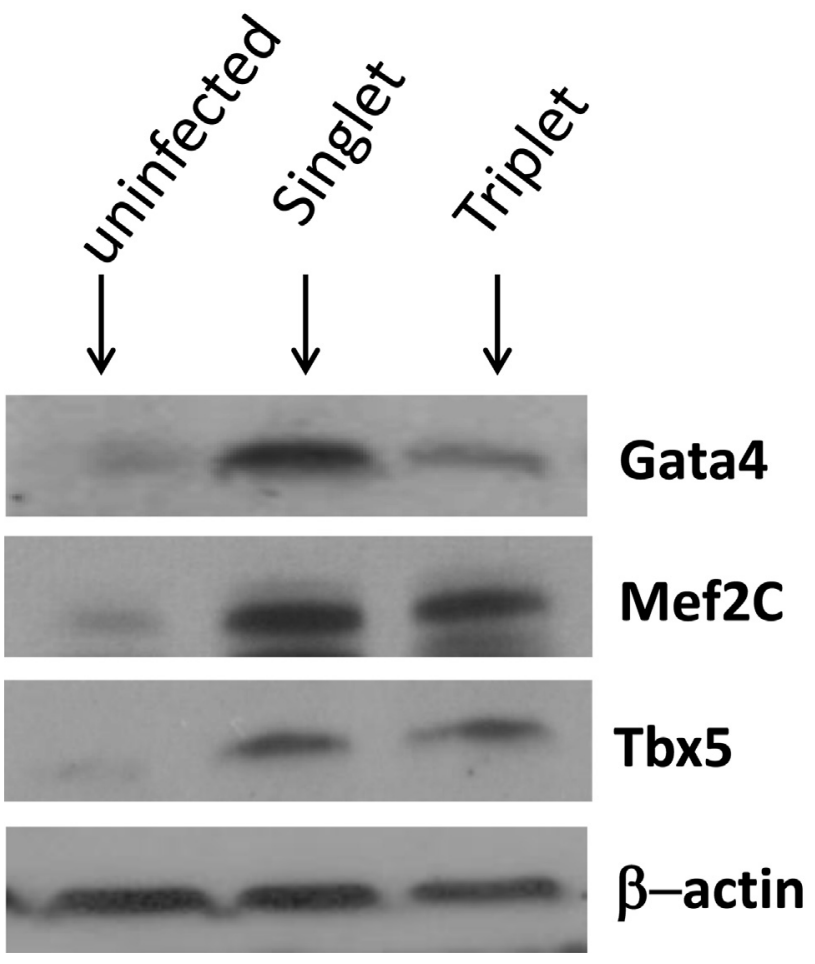

FIGURE E3. Western blot assay of in vitro culture cells. Fischer rat cardiac fibroblasts were infected with indicated viruses for 3 days, as described in the "Methods" section, and Western blots performed. Equivalent expression of Gata4, Mef2c, and Tbx5 is demonstrated for singlet Gata4, Mef2c, and Tbx5 (GMT) versus polycistronic triplet GMT lentivirus vector. 
TABLE E1. Primers used for plasmid construction

Primer name

Melting temperature $\left({ }^{\circ} \mathrm{C}\right)$

Gata4 5'

GGCGGTCGACATGTACCAAAGCCTGGCTATG

72.6

Gata4 3'

GATTCTCGAGTACGCGGTGATTATGTCCCCATG

70.9

Mef2c $5^{\prime}$

GGACTGTCGACATGGGGAGAAAAAAGATTCAG

68.5

Mef2c $3^{\prime}$

TGTGACTCGAGTCATGTTGCCCATCCTTCAGAGAG

71.7

$\operatorname{Tbx} 55^{\prime}$

CACCGTCGACATGGCCGACGCAGATGAG

73.4

Tbx $53^{\prime}$

CCTTCTCGAGTCAAGCTATTCTCGCTCCACTCTG

71.9 